\title{
Neutrinoless double-beta decay search with CUORE and CUORE-0 experi- ments
}

N. Moggi $i^{1,2, a}$, D. R. Artusa ${ }^{3,4}$, F. T. Avignone $I I^{3}$, O. Azzolini ${ }^{5}$, M. Balata ${ }^{4}$, T. I. Banks ${ }^{6,7}$, G. Bari $^{2}$, J. Beeman $^{8}$, F. Bellini ${ }^{9,10}$, A. Bersani ${ }^{11}$, M. Biassoni ${ }^{12,13}$, C. Brofferio ${ }^{12,13}$, C. Bucci ${ }^{4}$, X. Z. Cai ${ }^{14}$, A. Camacho ${ }^{5}$, A. Caminata $^{15}$, L. Canonica ${ }^{4}$, X. G. Cao ${ }^{14}$, S. Capelli ${ }^{12,13}$, L. Cappelli ${ }^{4,16}$, L. Carbone ${ }^{12}$, L. Cardani ${ }^{9,10}$, N. Casali ${ }^{4,17}$, L. Cassina ${ }^{12,13}$, D. Chiesa ${ }^{12,13}$, N. Chott ${ }^{3}$, M. Clemenza ${ }^{12,13}$, S. Copello ${ }^{15}$, C. Cosmelli9,10, O. Cremonesi ${ }^{13}$, R. J. Creswick ${ }^{3}$, J. S. Cushman ${ }^{18}$, I. Dafinei ${ }^{10}$, A. Dally ${ }^{19}$, V. Datskov ${ }^{13}$, S. Dell'oro ${ }^{4,20}$, M. M. Deninno ${ }^{2}$ S. Di Domizio ${ }^{15,11}$, M. L. Di Vacri' ${ }^{4,17}$, A. Drobizhev6, L. Ejzak ${ }^{19}$, D. Q. Fang ${ }^{14}$, H. A. Farach ${ }^{3}$, M. Faverzani ${ }^{12,13}$, G. Fernandes ${ }^{15,11}$, E. Ferri ${ }^{12,13}$, F. Ferroni ${ }^{9,10}$, E. Fiorini ${ }^{12,13}$, M. A. Franceschi ${ }^{21}$, S. J. Freedman ${ }^{6,7, b}$, B. K. Fujikawa ${ }^{7}$, A. Giachero ${ }^{12,13}$, L. Gironi ${ }^{12,13}$, A. Giuliani22 ${ }^{22}$ P. Gorla ${ }^{4}$, C. Gotti ${ }^{12,13}$, T. D. Gutierrez ${ }^{23}$, E. E. Haller ${ }^{8,24}$, K. Han ${ }^{18}$, K. M. Heeger ${ }^{18}$, R. Hennings-Yeomans ${ }^{6,7}$, K. P. Hickerson ${ }^{25}$, H. Z. Huang ${ }^{25}$, R. Kadel ${ }^{26}$, G. Keppel ${ }^{5}$, Yu. G. Kolomensky ${ }^{6,26}$, Y. L. Li $^{14}$, C. Ligi' ${ }^{21}$, K. E. Lim ${ }^{18}$, X. Liü25, Y. G. Ma ${ }^{14}$, C. Maiano ${ }^{12,13}$, M. Maino ${ }^{12,13}$, M. Martinez ${ }^{27}$, R. H. Maruyama ${ }^{18}$, Y. Mei ${ }^{7}$, S. Morganti ${ }^{10}$, T. Napolitano ${ }^{21}$, S. Nisi ${ }^{4}$, C. Nones ${ }^{28}$, E. B. Norman ${ }^{29,30}$, A. Nucciotti ${ }^{12,13}$, T. O'Donnell ${ }^{6,7}$, F. Orio ${ }^{10}$, D. Orlandi ${ }^{4}$, J. L. Ouellet ${ }^{6,7}$, C. E. Pagliarone ${ }^{4,16}$, M. Pallavicini ${ }^{15,11}$, V. Palmieri ${ }^{5}$, L. Pattavina ${ }^{4}$, M. Pavan ${ }^{12,13}$, G. Pessina ${ }^{13}$, V. Pettinacci ${ }^{10}$, G. Piperno ${ }^{9,10}$, C. Pira ${ }^{5}$, S. Pirro ${ }^{4}$, S. Pozzi ${ }^{12,13}$, E. Previtali ${ }^{13}$, C. Rosenfeld ${ }^{6}$, C. Rusconi ${ }^{13}$, E. Sala ${ }^{12,13}$, S. Sangiorgio ${ }^{29}$, D. Santone ${ }^{4,17}$, N. D. Scielzo ${ }^{29}$, M. Sisti ${ }^{12,13}$, A. R. Smith ${ }^{7}$, L. Taffarello ${ }^{31}$, M. Tenconi ${ }^{22}$, F. Terranova ${ }^{12,13}$, W. D. $\operatorname{Tian}^{14}$, C. Tomei ${ }^{10}$, S. Trentalange ${ }^{25}$, G. Ventura ${ }^{32,33}$, M. Vignati ${ }^{9,10}$, B. S. Wang ${ }^{29,30}$, H. W. Wang ${ }^{14}$, L. Wielgus ${ }^{19}$, J. Wilson ${ }^{3}$, L. A. Winslow ${ }^{25}$, T. Wise ${ }^{18,19}$, A. Woodcraft ${ }^{34}$, L. Zanotti ${ }^{12,13}$, C. Zarra ${ }^{4}$, G. Q. Zhang ${ }^{14}$, B. X. Zhu ${ }^{25}$, and S. Zucchelli ${ }^{35,2}$

${ }^{1}$ Dipartimento di Scienze per la Qualità della Vita, Alma Mater Studiorum - Università di Bologna, I-47921 - Italy

${ }^{2}$ INFN - Sezione di Bologna, Bologna l-40127 - Italy

${ }^{3}$ Department of Physics and Astronomy, University of South Carolina, Columbia, SC 29208 - USA

${ }^{4}$ INFN - Laboratori Nazionali del Gran Sasso, Assergi (L'Aquila) I-67010 - Italy

${ }^{5}$ INFN - Laboratori Nazionali di Legnaro, Legnaro (Padova) I-35020 - Italy

${ }^{6}$ Department of Physics, University of California, Berkeley, CA 94720 - USA

${ }^{7}$ Nuclear Science Division, Lawrence Berkeley National Laboratory, Berkeley, CA 94720 - USA

${ }^{8}$ Materials Science Division, Lawrence Berkeley National Laboratory, Berkeley, CA 94720 - USA

${ }^{9}$ Dipartimento di Fisica, Sapienza Università di Roma, Roma l-00185 - Italy

${ }^{10}$ INFN - Sezione di Roma, Roma l-00185 - Italy

${ }^{11}$ INFN - Sezione di Genova, Genova I-16146 - Italy

${ }^{12}$ Dipartimento di Fisica, Università di Milano-Bicocca, Milano I-20126 - Italy

${ }^{13}$ INFN - Sezione di Milano Bicocca, Milano I-20126 - Italy

${ }^{14}$ Shanghai Institute of Applied Physics, Chinese Academy of Sciences, Shanghai 201800 - China

${ }^{15}$ Dipartimento di Fisica, Università di Genova, Genova I-16146 - Italy

${ }^{16}$ Dipartimento di Ingegneria Civile e Meccanica, Università degli Studi di Cassino e del Lazio Meridionale, Cassino I-03043 - Italy

${ }^{17}$ Dipartimento di Scienze Fisiche e Chimiche, Università dell'Aquila, L'Aquila I-67100 - Italy

${ }^{18}$ Department of Physics, Yale University, New Haven, CT 06520 - USA

${ }^{19}$ Department of Physics, University of Wisconsin, Madison, WI 53706 - USA

${ }^{20}$ INFN - Gran Sasso Science Institute, L'Aquila I-67100 - Italy

${ }^{21}$ INFN - Laboratori Nazionali di Frascati, Frascati (Roma) I-00044 - Italy

${ }^{22}$ Centre de Spectrométrie Nucléaire et de Spectrométrie de Masse, 91405 Orsay Campus - France

${ }^{23}$ Physics Department, California Polytechnic State University, San Luis Obispo, CA 93407 - USA

${ }^{24}$ Department of Materials Science and Engineering, University of California, Berkeley, CA 94720 - USA

${ }^{25}$ Department of Physics and Astronomy, University of California, Los Angeles, CA 90095 - USA

${ }^{26}$ Physics Division, Lawrence Berkeley National Laboratory, Berkeley, CA 94720 - USA

${ }^{27}$ Laboratorio de Fisica Nuclear y Astroparticulas, Universidad de Zaragoza, Zaragoza 50009 - Spain

${ }^{28}$ Service de Physique des Particules, CEA / Saclay, 91191 Gif-sur-Yvette - France

${ }^{29}$ Lawrence Livermore National Laboratory, Livermore, CA 94550 - USA

${ }^{30}$ Department of Nuclear Engineering, University of California, Berkeley, CA 94720 - USA

${ }^{31}$ INFN - Sezione di Padova, Padova I-35131 - Italy

${ }^{32}$ Dipartimento di Fisica, Università di Firenze, Firenze 1-50125 - Italy

${ }^{33}$ INFN - Sezione di Firenze, Firenze 1-50125 - Italy 
${ }^{34}$ SUPA, Institute for Astronomy, University of Edinburgh, Blackford Hill, Edinburgh EH9 3HJ - UK

${ }^{35}$ Dipartimento di Fisica e Astronomia, Alma Mater Studiorum - Università di Bologna, I-40127 - Italy

\begin{abstract}
The Cryogenic Underground Observatory for Rare Events (CUORE) is an upcoming experiment designed to search for the neutrinoless double-beta decays. Observation of the process would unambiguously establish that neutrinos are Majorana particles and provide information on their absolute mass scale hierarchy. CUORE is now under construction and will consist of an array of $988 \mathrm{TeO}_{2}$ crystal bolometers operated at 10 $\mathrm{mK}$, but the first tower (CUORE-0) is already taking data. The experimental techniques used will be presented as well as the preliminary CUORE-0 results. The current status of the full-mass experiment and its expected sensitivity will then be discussed.
\end{abstract}

\section{Introduction}

Since the discovery of neutrino oscillations the interest in neutrino physics has increased, but some crucial questions concerning the nature of neutrinos remain open: the ordering and the absolute scale of the masses of the three generations, the charge conjugation and the lepton number conservation properties. If neutrinos are Majorana particles that differ from antineutrinos only by helicity, an important consequence is that lepton number violation must occur. The process of neutrinoless double-beta decay $(0 v \beta \beta)$ has the potential to provide insights on all these issues with unprecedented sensitivity. In fact, $0 v \beta \beta$ is the most realistic process and, at present, the only practical mean of experimental investigation on these topics [1][2].

Observation of the $0 v \beta \beta$ process, that violates lepton number conservation, would demonstrate the Majorana nature of neutrinos. At the same time it would allow to set constraints on the absolute mass scale. It should be noted, however, that $0 v \beta \beta$ could also be mediated by some exotic mechanism that would spoil most of the information concerning the neutrino mass; nevertheless it would still be the only way to probe the Majorana nature of neutrinos.

\section{The neutrinoless double-beta decay process}

Double-beta decay $(2 v \beta \beta)$ is a rare spontaneous nuclear transition $(Z, A) \longrightarrow(Z+2, A)+2 e^{-}+2 v_{e}$ in which a parent nucleus decays to a daughter with a simultaneous emission of two electrons. Within the Standard Model this is an allowed 2nd-order weak process already observed in different isotopes with an even number of neutrons and protons where the single-beta decay is either energetically forbidden or kinematically suppressed. The measured half-lives are as high as $10^{18}-10^{21} \mathrm{y}$, see e.g. [3]. If neutrinos are Majorana particles, i.e. are identical to their own antiparticle, the $v_{e}$ from one single beta decay may be absorbed in the second beta decay vertex (through helicity flipping) which would result in a final state without neutrinos and a lepton number violation of two units: $(Z, A) \longrightarrow(Z+2, A)+2 e^{-}$.

Half-life limits have been set for several isotopes, no experimental evidence of $0 v \beta \beta$ has been found to date except for a controversial claim in ${ }^{76} \mathrm{Ge}$ [7] which is hardly

\footnotetext{
a e-mail: niccolo.moggi@bo.infn.it

${ }^{\mathrm{b}}$ Deceased
}

compatible with more recent results, see for example [810]. At present, a combination of results in ${ }^{76} \mathrm{Ge}$ yields $T_{1 / 2}^{0 v}>3.0 \cdot 10^{25}$ y (90\% C.L.).

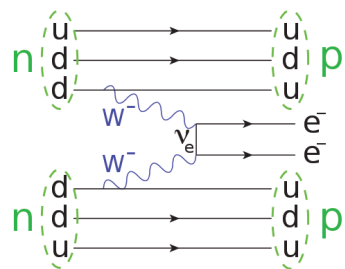

Figure 1. Feynman diagram of $0 v \beta \beta$.

\subsection{The decay rate}

The decay rate of the $0 v \beta \beta$ process is proportional to the square of the so-called effective Majorana mass $\left\langle m_{\beta \beta}\right\rangle$ and can be expressed as:

$$
\frac{1}{T_{1 / 2}^{0 v}}=G^{0 v}(Q, Z)\left|M^{0 v}\right|^{2} \frac{\left|\left\langle m_{\beta \beta}\right\rangle\right|^{2}}{m_{e^{-}}^{2}}
$$

where $G^{0 v}(Q, Z)$ is the phase-space factor (which can be calculated); $M^{0 v}$ is the transition nuclear matrix element (which also can be calculated, but different models may disagree by a factor of two to three (see, e.g., [5]); and $m_{e^{-}}^{2}$ is the electron mass. $\left\langle m_{\beta \beta}\right\rangle$ measure a specific mixture of neutrino mass eigenvalues:

$$
\left\langle m_{\beta \beta}\right\rangle=f\left(\theta_{12}, \theta_{23}, \theta_{13}, \Delta m_{12}, \pm \Delta m_{23}, m_{0}\right)
$$

Therefore form the half-life is possible to infer important information concerning the mass hierarchy $\left(\Delta m_{12}, \pm \Delta m_{23}\right)$ and the neutrino absolute mass scale $\left(m_{0}\right)$.

Present data from neutrino oscillation experiments tend to favour a range of $\left\langle m_{\beta \beta}\right\rangle$ values between 10 and 50 $\mathrm{meV}$ for the inverted hierarchy and roughly a factor 10 smaller for normal hierarchy $[1,6]$.

\section{Experimental approach}

\subsection{Signature}

A convenient experimental signature is given by the combined energy of the two final state electrons that are emitted simultaneously. Since the nucleus is heavy enough that 


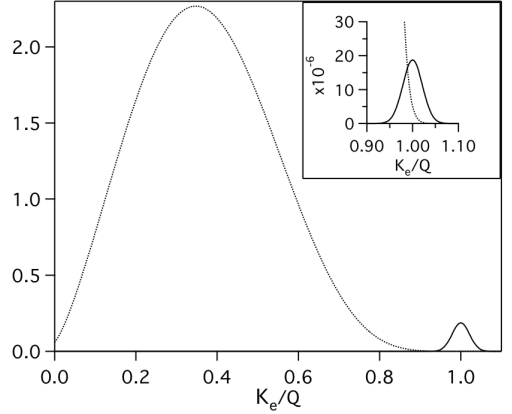

Figure 2. Energy spectrum of the electrons of the $2 v \beta \beta$ and $0 v \beta \beta$ decays.

all the energy is shared between the two electrons and the recoil is negligible, the $0 v \beta \beta$ decay signature would be a monochromatic line at the transition energy (Q-value) of the decay, while for the $2 v \beta \beta$ process a continuum spectrum between 0 and the Q-value would be observed. In both cases the distribution is smeared by the finite energy resolution of the detector and the tail of the $2 v \beta \beta$ distribution may overlap the $0 v \beta \beta$ peak, fig. 2. Should a $0 v \beta \beta$ peak be observed, the half-life could be estimated as:

$$
T_{1 / 2}^{0 v}=\ln (2) T \varepsilon \frac{N_{\beta \beta}}{N_{\text {peak }}}
$$

where $T$ is the time duration of the measure, $\varepsilon$ the detection efficiency, $N_{\beta \beta}$ the number of source nuclei, and $N_{\text {peak }}$ the number of observed $0 v \beta \beta$ decays.

\subsection{Bolometric technique}

CUORE (Cryogenic Underground Observatory for Rare Events) will use $\mathrm{TeO}_{2}$ crystals as bolometers to search for $0 v \beta \beta$ decay of ${ }^{130} \mathrm{Te}$. This technique was proposed by $\mathrm{E}$. Fiorini and T.O. Niinikoski in 1984 [11]. A bolometer is a calorimeter composed of an energy absorber, in which the energy deposited by a particle is converted into phonons, and a sensor that converts thermal excitation (temperature rise) into a readable electric signal. In our experimental setup the $\mathrm{TeO}_{2}$ crystals contain the decay isotope $\left({ }^{130} \mathrm{Te}\right)$ and, at the same time, act as detectors (the absorber material).

The temperature rise $\Delta \mathrm{T}$ is related to the energy release $\Delta \mathrm{E}$ and can be written as $\Delta \mathrm{T}=\Delta \mathrm{E} / \mathrm{C}$ where $\mathrm{C}$ is the heat capacity of the bolometer. When the crystals are cooled down to very low temperatures (few $\mathrm{mK}$ ), $\mathrm{C}$ becomes so small that few $\mathrm{keV}$ deposited into the detector will produce a measurable temperature rise. At the projected base temperature of about $10 \mathrm{mK}$ the typical signal amplitude is $\Delta \mathrm{T}=\Delta \mathrm{E} \sim 10-20 \mu \mathrm{K} / \mathrm{MeV}$ [4]. The accumulated heat flows then to a heat bath through a thermal link so that the absorber returns to the base temperature (this is reached in less than $5 \mathrm{~s}$ ). The temperature rise resulting from a single nuclear decay is measured by a thermistor. A Neutron Transmutation Doped thermistor is glued on each crystal. Since the thermal response of bolometers vary with the

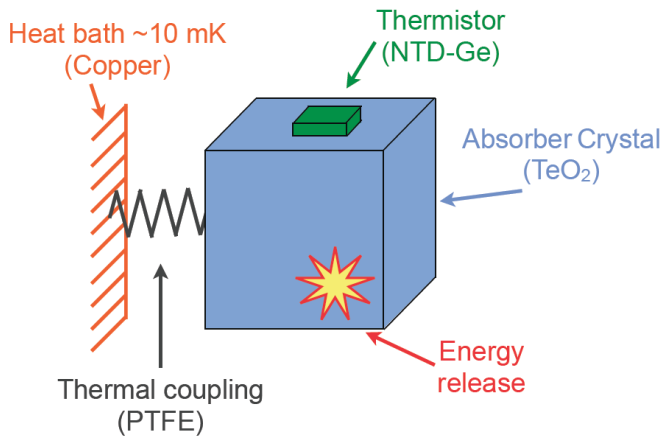

Figure 3. Schematic of a single CUORE-0 bolometer (not to scale).

temperature, a silicon Joule heater is glued to each crystal for the offline correction of thermal gain drift caused by thermal gain with time.

The energy resolution of a bolometer is limited, in principle, only by the thermodynamical fluctuations of thermal phonons through the thermal link, and does not depend on the deposited energy $\Delta \mathrm{E}$. In practice, $\Delta \mathrm{E}$ is dominated by other noise contributions; still, by using large mass bolometers, an excellent energy resolution and high detection efficiency can be achieved. Bolometers have also the advantage that they can be built with a wide range of materials, so several isotopes could be studied with this technique. The main drawbacks are that the thermal origin of the signal makes them intrinsically slow and that no event topology recognition is possible.

\subsection{Sensitivity}

For our kind of experimental setup, the sensitivity can be computed from simple arguments. The expected number of $0 v \beta \beta$ events (mean value), in a period $\mathrm{T}$ of time, is

$$
S \propto \text { (i.a.) } M \varepsilon \frac{T}{T_{1 / 2}^{0 v}}
$$

where (i.a.) is the isotopic abundance of the decay isotope, $\mathrm{M}$ the overall active mass and $\varepsilon$ the detector efficiency. In the same time period, for any experiment in which the source is embedded in the detector, the background B is given by:

$$
B \approx b M T \Delta E
$$

where $b$ is the background rate per unit detector mass (counts/(keV kg y)), and the energy window of the measure was approximated with the energy resolution $\Delta \mathrm{E}$ [13]. Given the background, the discovery potential of the experiment is given by the minimum signal counts that allow to reject the background-only hypothesis at a given significance $n_{\sigma}$ given in terms of gaussian standard deviations $n_{\sigma}=S / \sqrt{B}$. The discovery potential (sensitivity) can be then expressed in terms of the $0 v \beta \beta$ half-life, for a given significance $n_{\sigma}$, as:

$$
\widehat{T_{1 / 2}^{0 v}} \propto \frac{(\text { i.a. }) \varepsilon}{n_{\sigma}} \sqrt{\frac{M T}{b \Delta E}} \sim \sqrt{\frac{\text { detector scale }}{\text { performance }}}
$$



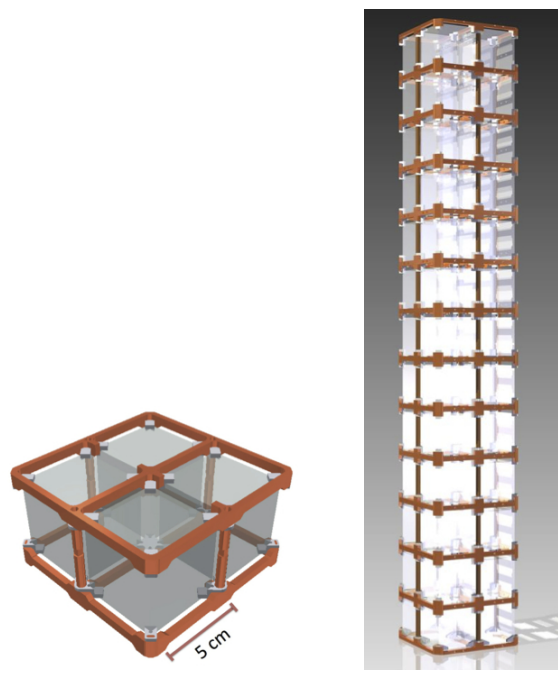

Figure 4. Sketch of a tower floor with 4 crystals (left). A single CUORE tower (right).

which links the sensitivity to the detector parameters. These parameters will be discussed in par. 4 .

Expression 6 holds as long as the number of background events is large enough to be considered gaussian. For low background experiments Poisson statistics should be used and, in the limit of zero background, the expression for the sensitivity would change into: $\widehat{T_{1 / 2}^{0 v}} \propto$ (i.a.) $\varepsilon T M$ where the sensitivity scales linearly with the detector mass.

\subsection{A phased search program}

The CUORE collaboration is going through a phased search program at the underground Gran Sasso National Laboratories where the flux of cosmic radiation is strongly reduced with respect to the sea level. Such program begun in 2003 with the Cuoricino experiment [12] (ended in 2008). The program resumed in 2013 with the CUORE-0 experiment built to demonstrate the feasibility of a largescale bolometric experiment (CUORE) and its potentials, and to test the stringent procedures to be adopted in the assembly line and many design improvements. This program will continue with CUORE, the full-mass setup. All experiments share the same bolometric technique and are built on radio-pure $\mathrm{TeO}_{2}$ cubic crystals. The crystals are $5 \times 5 \times 5 \mathrm{~cm}^{3}$ in size ${ }^{1}$ arranged in a compact array structure ("tower"), each floor consisting of 4 crystals.

\section{Detector parameters}

\subsection{Isotopic abundance and detector mass}

The choice of Tellurium is due to its high natural isotopic abundance (34.2\%) of the $0 v \beta \beta$ decay candidate [14]. Also, the Q-value around $2528 \mathrm{keV}$ of the decay [15-17] falls in a relatively clean window in which to look for the

\footnotetext{
${ }^{1}$ Cuoricino tested also some smaller crystals.
}

signal, between the peak and the Compton edge of the $2615 \mathrm{keV}$ gamma line of ${ }^{208} \mathrm{Tl}$.

Cuoricino and CUORE-0 have 62 and 52 crystals respectively, with roughly the same detector mass of about $40 \mathrm{~kg}$, organised in a single tower enclosed in a copper thermal shield and installed in the (same) cryostat. Cuoricino set the current lower limit for the half-life of ${ }^{130} \mathrm{Te}$ to $T_{1 / 2}^{0 v}>2.8 \cdot 10^{24}$ y (90\% C.L.) [12]. CUORE-0, which is now taking data, was assembled with the same materials as CUORE, and according to the same radiopurity constraints imposed on all the materials facing the detectors and on the detectors themselves to reduce the background sources. Therefore it represents an opportunity to evaluate the bolometric performance of CUORE but, as a standalone experiment, will be able to produce an improvement with respect to the Cuoricino results.

CUORE will consist of 988 crystals arranged in 19 towers. The total detector mass will be $741 \mathrm{~kg}$ and the ${ }^{130} \mathrm{Te}$ isotope mass is $206 \mathrm{~kg}$. It was designed to search for $0 v \beta \beta$ in ${ }^{130} \mathrm{Te}$ with the best sensitivity to date and will contribute in demonstrating the viability of future large-scale bolometric experiments.

\subsection{Background}

The background is the main limit to the CUORE sensitivity. To accomplish its suppression the first step was to identify the main sources of background in Cuoricino. The dominant source $(50 \pm 20) \%$ in the energy region of interest (ROI, around the $\mathrm{Q}$-value) was found to be from $\alpha$ particles emitted by contaminations of ${ }^{238} \mathrm{U},{ }^{232} \mathrm{Th},{ }^{210} \mathrm{~Pb}$ present on the surface of the copper parts that hold the crystals and of the materials facing the bolometers. Another $(10 \pm 5) \%$ was due to $\alpha$ from the same contaminants on the bolometer surfaces. Both these contribution are reduced in CUORE-0 with respect to Cuoricino thanks to the controlled construction materials and to the new dedicated surface-cleaning procedures developed for the handling and cleaning of each detector component. The cleaning procedure for the copper frames, in particular, was verified with a dedicated bolometric measurement [18]. Above the $2615 \mathrm{keV}^{208} \mathrm{Tl}$ peak the $\gamma$ background becomes negligible and the $\alpha$ background dominates (fig.5).

The second largest source $(30 \pm 10) \%$ was due to the $\gamma$ from ${ }^{208} \mathrm{Tl}$ originated by the decay chain of the ${ }^{232} \mathrm{Th}$ contamination in the cryostat materials. In CUORE this component is expected to be negligible thanks to the better shielding of the detector from the cryostat.

The background due to cosmics and environmental radiation in ROI is orders of magnitude smaller than that from the apparatus itself thanks to a combination of the underground location and several shielding layers both outside and inside the cryostat. One of the shielding layers is made of lead bricks recovered from an ancient roman ship sunk offshore the Sardinia coast around year 50 b.c. [19].

Finally, the tail from $2 v \beta \beta$ is also negligible thanks to the excellent energy resolution.

The CUORE design background is 0.01 counts/(kev kg y). The overall backgrounds in ROI is reported in table 1 . 


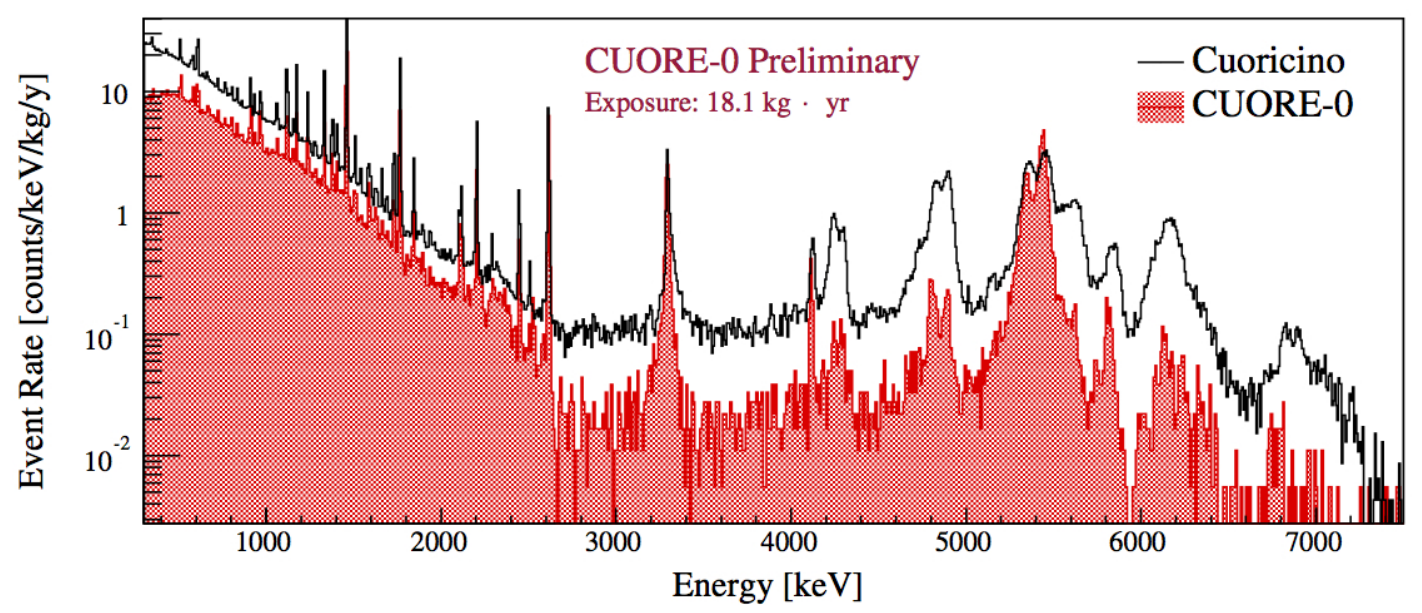

Figure 5. Background energy spectrum in Cuoricino and CUORE-0.

\subsection{Energy Resolution}

The energy resolution in the ROI was evaluated, with the CUORE-0 first phase data, as the FWHM of the $2615 \mathrm{keV}$ photo peak originated by the ${ }^{208} \mathrm{Tl}$ decay (which is close to the Q-value of $2527.5 \mathrm{keV}$ ). The overall detector resolution was found to be $5.7 \mathrm{keV}$ [20]. During some R\&D runs the target energy resolution of $5 \mathrm{keV}$ was consistently achieved [4], and in phase II of CUORE-0 reached $4.8 \mathrm{keV}$. It should also be noted that the R\&D tests and CUORE-0 run at a base temperature of about $13 \mathrm{mK}$, higher than the CUORE projected base temperature of 10 $\mathrm{mK}$. In conclusion it is expected that the CUORE performance will reach the project value of $\Delta \mathrm{E} \simeq 5 \mathrm{keV}$ or better.

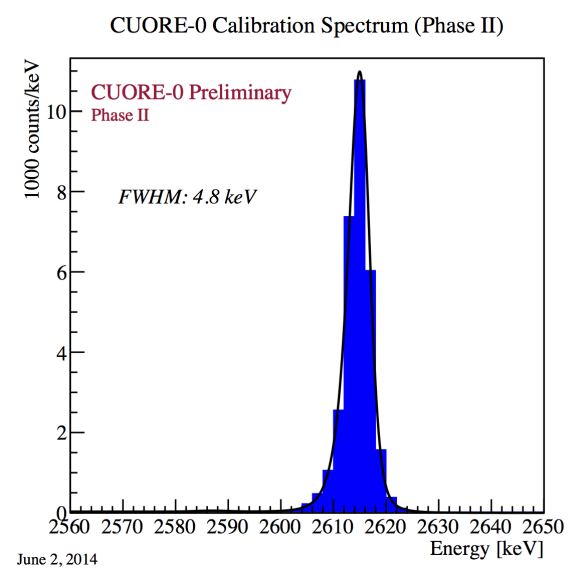

Figure 6. The ${ }^{208} \mathrm{Tl}$ decay line used to estimate the energy resolution in CUORE-0.

\subsection{Efficiency}

The selection efficiency is evaluated mainly using the 2615 $\mathrm{keV} \gamma$ peak which offers sufficient statistics at the energy closest to the ROI. The physical detector efficiency alone
Table 1. Total background in ROI and in the $\alpha$ region, in counts/(keV kg y). For CUORE the predicted value is reported.

\begin{tabular}{l|c|c}
\hline & $0 v \beta \beta$ & $2700-3900 \mathrm{keV}$ \\
\hline Cuoricino & $0.153 \pm 0.006$ & $0.110 \pm 0.001$ \\
CUORE-0 & $0.063 \pm 0.006$ & $0.020 \pm 0.001$ \\
CUORE & 0.01 & \\
\hline
\end{tabular}

is estimated to be $(87.4 \pm 1.1) \%$ and represents the containment of the detector to double-beta decay signals. By including the trigger and selection efficiencies (see par. 5) the total $0 v \beta \beta$ detection efficiency of CUORE- 0 is estimated to be $(77.6 \pm 1.3) \%$.

\section{CUORE-0 Preliminary Results}

CUORE-0 is operated in the same cryostat, uses the same external shielding, and is enclosed in the same Faraday cage that was used for Cuoricino. The front-end electronics and the data acquisition hardware are also the same. The offline data analysis follows the procedure developed for Cuoricino [12, 21]. Each bolometer has an independent

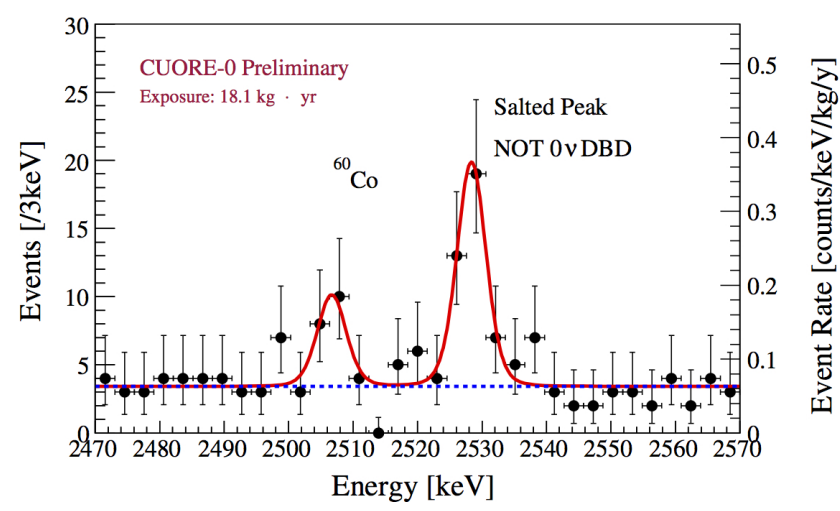

Figure 7. CUORE-0 energy spectrum: preliminary results of data taking phases I and II. 
signal trigger threshold and is pulsed periodically with a fixed, known energy through the heater. The pulsed energy events are used to correct for small shifts in thermal gain of the bolometers. Bolometer signals are amplified, filtered, digitized and then converted into energies using calibration data. Each channel is periodically calibrated using $\gamma$ rays from daughter nuclei of ${ }^{232} \mathrm{Th}$ in the energy range from 511 to $2615 \mathrm{keV}$. Events occurring within $\pm 100 \mathrm{~ms}$ of each other in any two or more crystals are attributed to background and therefore rejected. The pile-up selection cut requires that only one pulse exists in a $7.1 \mathrm{~s}$ window around the measured trigger time. Subsequent selections impose some shape requirements on the signal pulses.

CUORE-0 has been taking data in stable operating conditions from March to September 2013 ("phase I") and from November 2013 to June 2014 ("phase II"). A third phase is now going on. Phase I data were published in [20]; the preliminary results shown in fig. 7 refer to the data collected during phases I and II. The accumulated $\mathrm{TeO}_{2}$ exposure on 49 fully active channels is $18.06 \mathrm{~kg} \cdot \mathrm{y}$ for a ${ }^{130} \mathrm{Te}$ isotopic exposure of $5.02 \mathrm{~kg} \cdot \mathrm{y}$.

At present the data in the ROI is blinded while more statistics is being accumulated and the event selection is optimised. A blinded fraction of events within $\pm 10 \mathrm{keV}$ of the $2615 \mathrm{keV} \gamma$ peak is randomly exchanged with events within $\pm 10 \mathrm{keV}$ of the $0 v \beta \beta$ Q-value. Since the number of $2615 \mathrm{keV} \gamma$ events is much larger than the number of possible $0 v \beta \beta$ events, the blinding algorithm produces an artificial peak around the $0 v \beta \beta$ Q-value.

CUORE-0 already demonstrated the feasibility of instrumenting an ultra-pure ton-scale bolometer array like CUORE.

\section{CUORE}

\subsection{Constructions status}

While CUORE-0 is taking data, the collaboration is building the full-mass scale experiment, CUORE (fig. 8), with the goal to begin data taking in the summer of 2015. The assembly process consists in four main steps. Thermistors and heathers are glued to the crystals, the instrumented crystals are assembled together into a single tower, the readout cables are attached to the tower, for each crystal the thermistor and heather chips are bonded to the readout cable trays. All the assembling procedures are performed in glove boxes flushed with nitrogen in an underground clean room with custom designed tools that make the whole procedure semi-automatic.

At present, all 19 towers have been assembled, instrumented, and stored in nitrogen-flushed atmosphere while waiting to be installed in the cryostat (fig. 9). The collaboration is now moving toward detector integration, which includes the commissioning of the new cryostat, the installation of the calibration system, data acquisition system, faraday cage and other auxiliary systems like the slow control and monitoring system.

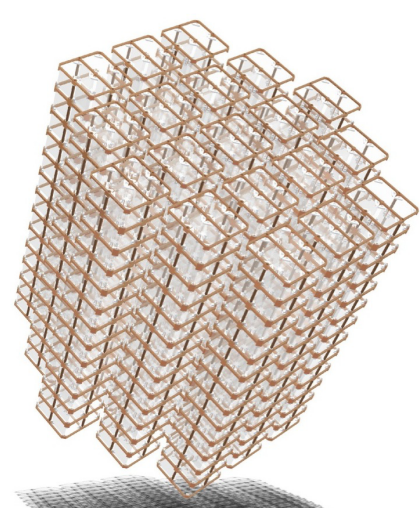

Figure 8. Sketch of the 988 CUORE bolometer array.

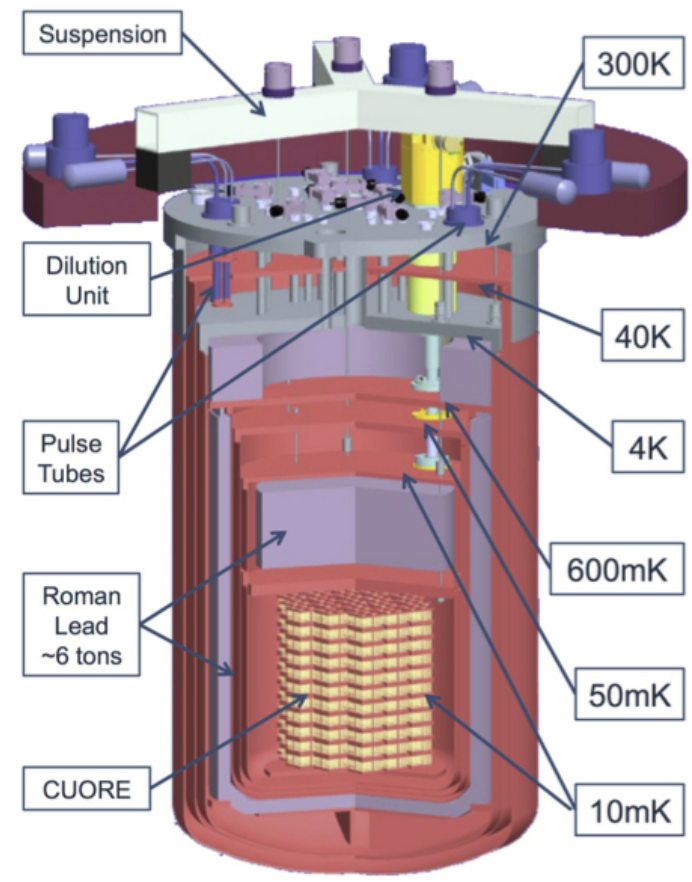

Figure 9. The CUORE cryostat, with major components highlighted.

\subsection{Projected sensitivity}

The closely-packed CUORE detector geometry will carry some benefits with respect to CUORE-0. First, it will enable a significant improvement in the anti coincidence analysis between close crystals, further reducing the background. Second, the fraction of crystals facing the contaminated inner shield will be reduced. Additionally, the new cryostat is built with cleaner materials and better shielded.

CUORE-0 has demonstrated that the CUORE design parameters of equation 6 reported in table 2, in particular the total mass, the background rate level and the energy resolution, can be reached. With such parameters, it is possible to compute the final sensitivity of CUORE as a function of the live-time. An overview of the $1 \sigma$ sensitivity of CUORE-0 and CUORE is shown in fig. 10. A half-life sensitivity close to $10^{25}$ years is expected for a 


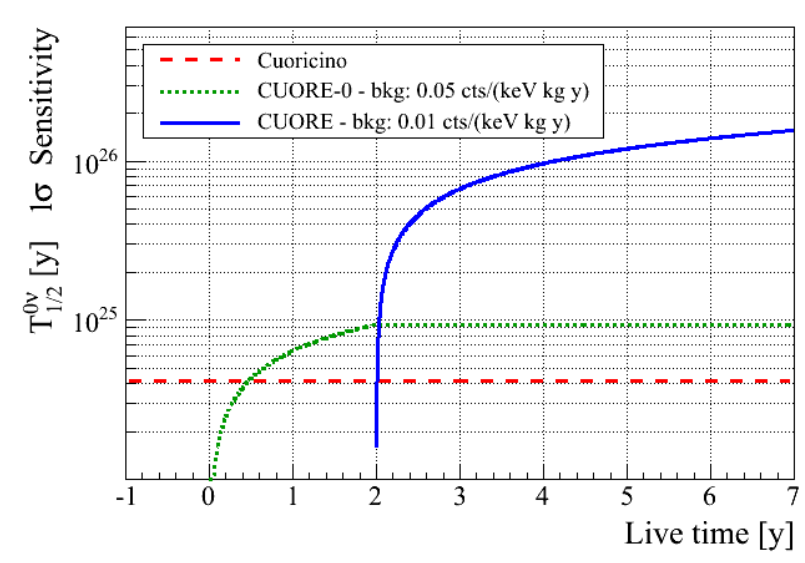

Figure 10. $1 \sigma$ sensitivity for CUORE-0 and CUORE computed using the experimental parameters given in table 2 .

2 years live-time of CUORE-0 after which CUORE will begin data taking.

It is possible, with equation 1 , to express the half-life sensitivity of equation 6 as sensitivity on the effective Majorana mass $\left\langle m_{\beta \beta}\right\rangle$. In fig. 11 the expected CUORE $1 \sigma$ sensitivity to $\left\langle m_{\beta \beta}\right\rangle$ is shown overlaid on the bands preferred by neutrino oscillation data for normal and inverted hierarchy, as a function of the lightest neutrino mass. The width of the CUORE band corresponds to the maximum and minimum $\left\langle m_{\beta \beta}\right\rangle$ values obtained from different nuclear matrix elements.

CUORE will fully explore the half-life corresponding to the claim of observation in ${ }^{76} \mathrm{Ge}$ and will allow the investigation of the upper region of the effective Majorana neutrino mass phase space for inverted hierarchy of neutrino masses.

\subsection{Beyond CUORE}

Next generation experiments should be able to explore the whole inverted hierarchy region of effective Majorana masses. Should the next generation experiments fail in finding $0 v \beta \beta$, it may still be possible, thanks to the input from other experiments, to draw some conclusions on the nature of neutrinos: if neutrino is proven to be a Majorana particle, then the mass hierarchy would have to be normal; on the other hand, if the mass hierarchy is proven to be inverted, then the neutrino would have to be a Dirac particle.

Since it is unlikely that CUORE itself will be able to

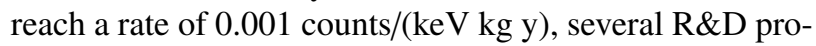
grams are already underway investigating new ideas and techniques for active background rejection.

Table 2. Experimental parameter values used for the sensitivity of CUORE-0 and CUORE. Symbols are defined in equation 6.

\begin{tabular}{l|c|c|c|c|c}
\hline & i.a. & $\begin{array}{c}\varepsilon \\
(\%)\end{array}$ & $\begin{array}{c}\mathrm{M} \\
(\mathrm{kg})\end{array}$ & $\begin{array}{c}\Delta \mathrm{E} \\
(\mathrm{keV})\end{array}$ & $\begin{array}{c}\mathrm{b} \\
\left(\frac{c t s}{k e V k g y}\right)\end{array}$ \\
\hline CUORE-0 & 34.167 & 87.4 & 39 & 5 & 0.05 \\
CUORE & 34.167 & 87.4 & 741 & 5 & 0.01 \\
\hline
\end{tabular}

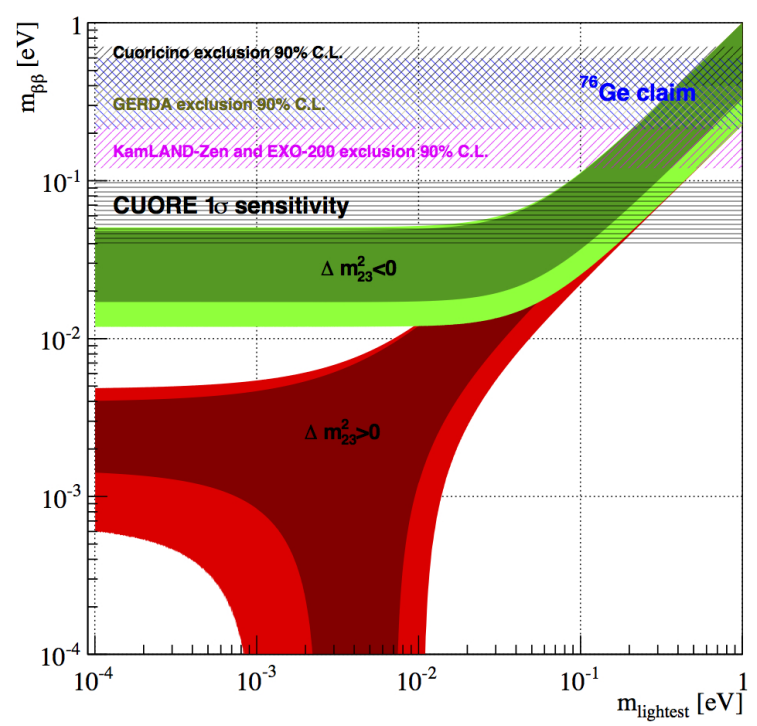

Figure 11. CUORE $1 \sigma$ sensitivity in terms of effective Majorana mass for 5 years live time computed using the experimental parameters given in table 2 . The bands corresponds to the maximum and minimum $\left\langle m_{\beta \beta}\right\rangle$ values obtained from different nuclear matrix elements.

In CUORE the sensitivity is mainly limited by background which is characterised by $\alpha$ and $\beta / \gamma$ emission in the $\mathrm{MeV}$ region. Particle identification is therefore the main emphasis on bolometers future application. To this end, additional detection channels are needed, since the absorber does not respond differently for energy releases of different particle types. To distinguish signal electrons from $\alpha$ background, light emission can be used, either from Cherenkov radiation [22, 23] or scintillation light $[24,25,28,29]$, where the auxiliary light detector is usually another bolometer facing the main one. Recently new studies on scintillating bolometers showed the possibility to distinguish $\alpha$ from $\beta / \gamma$ particles without light readout thanks to a different time-dependent shape of the heat signal [30, 31]. Alternative methods based on the identification of surface interactions have also been devised (see, e.g. $[26,27])$.

\section{Conclusions}

A brief introduction to the bolometric technique used to search for neutrinoless double-beta decay was given and the main experimental challenges outlined. The physics reach of CUORE-0 and CUORE was illustrated.

CUORE- 0 is at present the most sensitive experiment for searching $0 v \beta \beta$ in ${ }^{130} \mathrm{Te}$. Although it's still taking data, it has already confirmed that the design parameters of the full-size experiment, CUORE, can be reached, in particular with respect to the energy resolution and the background rate.

CUORE is now being built and it is expected that it will begin taking data in 2015 . With excellent energy resolution and large isotope mass, CUORE is one of the most competitive $0 v \beta \beta$ experiments under construction. The target background of 0.01 counts/( $\mathrm{keV} \mathrm{kg} \mathrm{y})$ seems within 
reach and in 5 years it is expected to achieve a $1 \sigma$ sensitivity to the half-life of ${ }^{130} \mathrm{Te}$ of $1.6 \times 10^{26}$ y $\left(9.5 \times 10^{25} \mathrm{y}\right.$ at $90 \%$ C.L.). In terms of effective Majorana mass, this corresponds to $1 \sigma$ sensitivity in the range of $40-100 \mathrm{meV}$ (50-130 $\mathrm{meV}$ at $90 \%$ C.L.).

\section{Acknowledgments}

The CUORE Collaboration thanks the directors and staff of the Laboratori Nazionali del Gran Sasso and the technical staff of our laboratories. This work was supported by the Istituto Nazionale di Fisica Nucleare (INFN); the Director, Office of Science, of the U.S. Department of Energy under Contract Nos. DE-AC02-05CH11231 and DE-AC52-07NA27344; the DOE Office of $\mathrm{Nu}$ clear Physics under Contract Nos. DE-FG02-08ER41551 and DEFG03-00ER41138; the National Science Foundation under Grant Nos. NSF-PHY-0605119, NSF-PHY0500337, NSF-PHY-0855314, NSF-PHY-0902171, and NSF-PHY-0969852; the Alfred P. Sloan Foundation; the University of Wisconsin Foundation; and Yale University. This research used resources of the National Energy Research Scientific Computing Center (NERSC).

\section{References}

[1] A. Strumia, F. Vissani, Neutrino masses and mixings and..., arXiv:hep-ph/0606054 (2010)

[2] A. deGouvea, P. Vogel, Lepton flavor and number conservation, and physics beyond the standard model, arXiv:1303.4097 (2013)

[3] R. Arnold et al., Phys. Rev. Lett. 107, 062504 (2011)

[4] D.R. Artusa et al., Searching for neutrinoless doublebeta decay of ${ }^{130} \mathrm{Te}$ with CUORE, accepted by Adv. High Energy Physics (2014), axXiv:1402.6072

[5] S.M. Bilenky, C. Giunti, Neutrinoless double-beta decay. A brief review., Mod. Phys. Lett. A27 (2012) 1230015, arXiv: 1203.5250

[6] X. Sarazin, Review of double-beta experiments, arXiv:1210.7666, 2012

[7] H.V. Klapdor-Kleingrothaus, International Journal of Modern Physics E 17 (2008) 505-517

[8] M. Auger et al. (EXO Coll.) Phys.Rev.Lett 109, 032505 (2012)

[9] A. Gando et al. (KamLAND-Zen Coll.) Phys.Rev.Lett. 110062502 (2013)

[10] M. Agostini et al. (GERDA Coll.) Phys.Rev.Lett 111 22503 (2013)

[11] E. Fiorini, T.O. Niinikoski, Nucl. Instrum. Meth. A 224, 83 (1984)

[12] E. Andreotti et al. (CUORICINO Coll.), ${ }^{130} \mathrm{Te} \mathrm{Neu}-$ trinoless Double-Beta Decay with CUORICINO, Astropart. Phys. 34 (2011) 822-831.
[13] F. Alessandria et al., Sensitivity of CUORE to Neutrinoless Double-Beta Decay, submitted to Astropart. Physics (2014) arXiv:1109.0494.

[14] M.A. Fehr et al., Int. J. Mass Spect. 232, 83 (2004)

[15] M. Redshaw et al., Phys. Rev. Lett. 102, 212502 (2009), arXiv:0902.2139

[16] N.D. Scielzo et al., Phys. Rev. C 80, 025501 (2009), arXiv:0902.2376

[17] S. Rahaman et al., Phys. Lett. B 703, 412 (2011)

[18] F. Alessandria et al., Validation of techniques to mitigate copper surface contamination in CUORE, Astropart.Phys. 45, 13 (2013), arXiv:1210.1107

[19] A. Alessandrello et al., Measurements of internal radioactive contamination in samples of Roman Lead to be used in experiments on rare events, Nucl. Instr. Meth. B 142 (1998) 163

[20] D.R. Artusa et al., Initial performance of the CUORE-0 experiment, Eur.Phys.J. C 74 (2014) 2956, arXiv: 1402.0922

[21] C. Arnaboldi et al., Results from the CUORICINO neutrinoless double beta decay experiment, Phys. Rev. C 78 (2008) 035502, arXiv:0802.3439

[22] T. Tabarelli de Fatis, Cherenkov emission as a positive tag of double beta decays in bolometric experiments, Eur. Phys. J. C 65 (2010) 359

[23] J. Beeman et al., Discrimination of alpha and beta/gamma interactions in a $\mathrm{TeO} 2$ bolometer, Astropart. Phys. 35 (2012) 558, arXiv:1106.6286.

[24] G. Angloher et al.,Results from $730 \mathrm{~kg}$ days of the CRESST-II Dark Matter Search, Eur. Phys. J. C 72 (2012) 1971, arXiv:1109.0702.

[25] S. Domizio et al., Cryogenic wide-area light detectors for neutrino and dark matter searches, J. Low. Temp. Phys. (2014) 1-7 40 C.

[26] C. Isaila et al., Low-temperature light detectors: Neganov-Luke amplification and calibration, Phys. Lett. B 716 (1) (2012)

[27] M. Willers et al., Background discrimination in neutrinoless double beta decay search with $\mathrm{TeO}_{2}$ bolometers using Neganov-Luke amplified cryogenic light detectors, arXiv:1407.6516.

[28] S. Pirro,et al., Scintillating double beta decay bolometers, Phys. Atom. Nucl. 69 (2006) 2109.

[29] C. Arnaboldi et al., Characterizations of ZnSe scintillating bolometers for Double Beta Decay, Astropart. Phys. 34 (2011) 344

[30] J. Beeman et al., Performance of a large mass $\mathrm{ZnMoO}_{4}$ scintillating bolometer for a next generation neutrinoless double beta decay experiment, Eur. Phys. J, C 72 (2012) 1

[31] J. Beeman et al., $\mathrm{ZnMoO}_{4}$ : A promising bolometer for neutrinoless double beta decay experiment, Astropart. Phys. 35813 (2012) 